\title{
Pituitary apoplexy in setting of Dengue Hemorrhagic Fever with thrombocytopenia: case report and review of literature
}

\author{
Abhay Singh ${ }^{1}$, Rahul Gupta ${ }^{2}$, Gangesh Gunjan ${ }^{3}$, Harjinder Singh \\ Bhatoe $^{4}$, Dhawal Sharma ${ }^{4}$ \\ ${ }^{1}$ General Surgery, Neurosurgery, Fortis Hospital, Noida, U.P., INDIA \\ ${ }^{2}$ Neurosurgery, Additional Director Neurosurgery, Fortis Hospital, Noida, U.P., INDIA \\ ${ }^{3}$ Consultant Neurosurgery, Fortis Hospital, Noida, U.P., INDIA \\ ${ }^{4}$ Neurosurgery, Fortis Hospital, Noida, U.P., INDIA
}

\begin{abstract}
Pituitary apoplexy is an acute clinical syndrome. It may occur spontaneously or as a result of several precipitating factors; one such factor being thrombocytopenia. Acute febrile illness accompanying with bleeding tendency is the main clinical feature of dengue. If the diagnosis is made in time, urgent treatment in the form of decompression of optic nerves may help to save vision. According to literature, only seven cases have been reported with pituitary apoplexy in setting of Dengue hemorrhagic fever. We report eighth case of Pituitary apoplexy in patient having Dengue hemorrhagic fever with its management and review of literature.
\end{abstract}

Key words: Dengue Hemorrhagic Fever, Pituitary apoplexy, Thrombocytopenia

\section{Introduction}

Pituitary apoplexy is an acute clinical syndrome characterized by sudden headache, vomiting, visual disturbances, ophthalmoplegia, altered consciousness, secondary to infarction or hemorrhage within a pituitary tumor or nontumorous pituitary gland. [1,2] The diagnosis is often missed in the early stages. CT and MRI of the brain are helpful in making the diagnosis of pituitary apoplexy. Pituitary apoplexy may occur spontaneously or as a result of several precipitating factors; one such factor being thrombocytopenia.

Dengue is an infectious disease which can be seen worldwide. Acute febrile illness accompanying with bleeding tendency is the main clinical feature of dengue. [3] Dengue hemorrhagic fever (DHF) is characterized by fever, hemorrhagic tendencies, thrombocytopenia, and increased vascular permeability. [4] According to literature, only seven cases have been reported with pituitary apoplexy in setting of Dengue hemorrhagic 
fever. We report eighth case of Pituitary apoplexy in the setting of Dengue hemorrhagic fever with its management and review of literature.

\section{Case report}

A 48 year old female presented with history of high grade fever 3 days. She was evaluated at local hospital where serology for Dengue infection was positive and she had low platelet count. Patient was referred to our hospital for further management. There was no history of petechial rash and spontaneous bleeding from mucosal surfaces. On third day of admission she complaint of sudden diminution of vision and severe headache. The platelet count decreased to 17,000. (Table 1)

Table 1 - Daily Platelet count

\begin{tabular}{|l|l|l|l|l|l|l|}
\hline $\begin{array}{l}\text { Platelets } \\
/ \mathrm{mm} 3)\end{array}$ & $\begin{array}{l}\text { On } \\
\text { Admission }\end{array}$ & Day 1 & Day 2 & Day 3 & Day 4 & Day 5 \\
\hline $\begin{array}{l}\text { Platelet } \\
\text { count }\end{array}$ & 72,000 & 50,000 & 22,000 & 17,000 & 33,000 & 131,000 \\
\hline
\end{tabular}

NCCT Head was done which was suggestive of presence of sellar/suprasellar well defined heterogeneously hyperdense mass $(25 \mathrm{~mm} \quad \mathrm{x} 20 \mathrm{~mm} \quad \mathrm{x} \quad 18 \mathrm{~mm})$ with hyperdense area of bleed. (Figure 1)

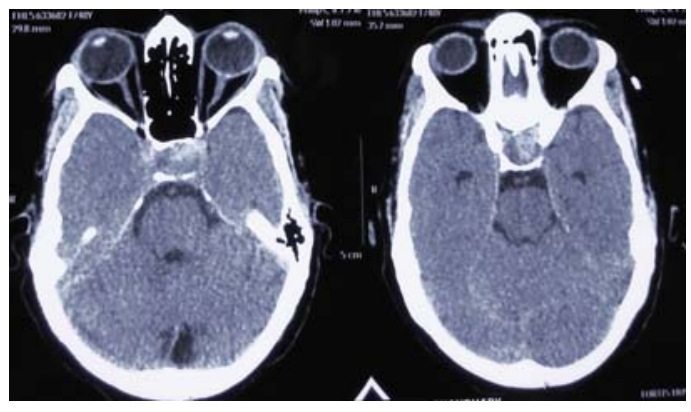

Figure 1- NCCT Head Axial sections: Sellar/suprasellar well defined heterogeneously hyperdense mass with hyperdense area of bleed

MRI brain showed well defined in homogeneously enhancing sellar- suprasellar mass with internal area of bleed. (Figure 2, $3 \& 4$ )

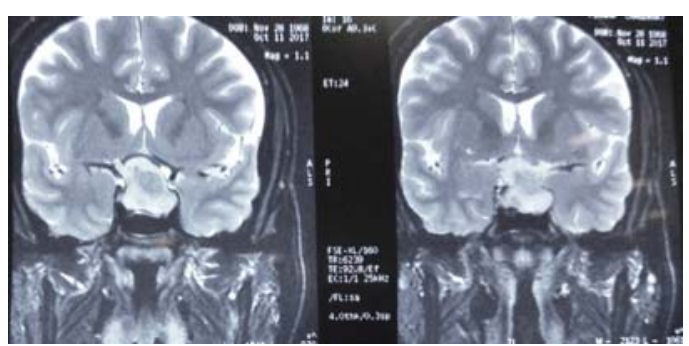

Figure 2 - MRI T2 WI Coronal Sections:

Heterogeneously hyperintense signal with presence of areas of bleed

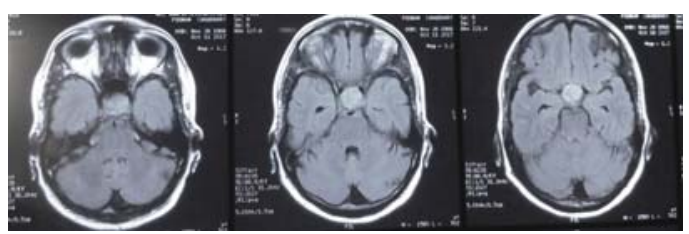

Figure 3- MRI FLAIR Axial sections: Well defined extraaxial mass in sellar / suprasellar region measuring $2.6 \times 2.3 \times 2 \mathrm{~cm}$ in size

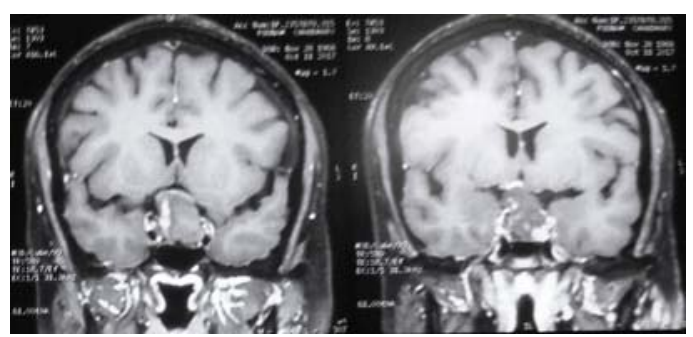

Figure 4 - MRI contrast enhanced Coronal sections: Mild to moderate inhomogenous post contrast enhancement with hemorrhagic area

The optic chiasm was draped on its superior surface with no visualized area of in 
between planes. On examination patient had Bitemporal hemianopia by confrontation method. Patient's hormone profile revealed decreased serum thyroid and prolactin hormone levels [Growth Hormone $(\mathrm{GH})=$ $2.30 \mathrm{ng} / \mathrm{mL}$; Luteinizing Hormone (LH) $=2.64 \mathrm{mIU} / \mathrm{mL}$; Prolactin $(\mathrm{PRL})=2.23 \mathrm{ng} / \mathrm{mL}$; Cortisol $=6.53 \mu / \mathrm{dL} ;$ Follicle Stimulating Hormone $=5.85 \mathrm{mIU} / \mathrm{mL}$; T3 $=47.87 \mathrm{ng} / \mathrm{dL}$; Thyroxine $(\mathrm{T} 4)=4.82 \mu \mathrm{g} / \mathrm{dL} ;$ Thyroid Stimulating Hormone $(\mathrm{TSH})=1.220$ $\mu \mathrm{IU} / \mathrm{mL})$ ]

Patient underwent several platelet units transfusion and was operated after platelet count reached above 100,000. Urgent Transnasal transsphenoidal decompression of the pituitary macroadenoma was performed. Intraoperatively, there was evidence of bleed inside the tumor Post operative period was uneventful and patient was discharged subsequently. Histopathology was consistent with Pituitary apoplexy with bleed. On three month follow up, patient had hypocortisolemia with no visual defect.

\section{Discussion}

Pituitary apoplexy may occur spontaneously or as a result of several precipitating factors such as head trauma, dynamic tests to evaluate pituitary function, surgery (mainly cardiac surgery), coagulation disorders, medications including aspirin, estrogens, heparin, and dopamine agonists and some conditions such as chronic systemic hypertension, diabetes mellitus, and radiotherapy. $[1,2,5,6]$

The precipitants of pituitary apoplexy can be classified into 4 categories: (1) reduced blood flow into the pituitary adenoma, resulting in infarction; (2) acute increase in blood flow to the pituitary gland from the hypothalamus portal system because it may increase the intra sellar pressure; (3) stimulation of the pituitary gland, as occurs in any stress inducing states; and (4) thrombocytopenia because of increased risk of bleeding. [5, 7]

Dengue fever is endemic in tropical countries and can range from a nonspecific febrile illness to DHF, which is characterized by high grade fever, marked thrombocytopenia $(<100,000 / \mu \mathrm{L})$, and increased vascular permeability. [4] In DHF, patients may have episodes of hypotension and hypovolemia, leading to oscillations in blood pressure. [4] Because it is an acute systemic illness, there is an increased demand on the pituitary gland and pituitary stimulation. In addition, DHF is characterized by severe thrombocytopenia and increased hemorrhagic tendencies. All these factors combined may have a compounding effect on the risk of pituitary apoplexy in a pre existing pituitary adenoma. However, the association of DHF and PA is exceedingly rare and only few case reports document their co occurrence. $[6,8,9,10,11$, 12] Other causes of visual deterioration in patients with dengue fever are optic neuropathy, maculopathy, retinal capillary occlusion, foveolitis, and retinal hemorrhage. $[8,13]$ The review of literature regarding the incidence of pituitary apoplexy in the setting of DHF revealed seven previously reported cases. The summary of these cases along with their comparative features has been described in Table 2. (Table 2) 
300 | Singh et al - Pituitary apoplexy in Dengue Hemorrhagic fever

Table 2 - Summary of the reported cases of pituitary apoplexy in setting of Dengue Hemorrhagic Fever

\begin{tabular}{|c|c|c|c|c|c|c|c|}
\hline Reference & \begin{tabular}{|l|} 
Age/ \\
Sex \\
\end{tabular} & \begin{tabular}{|l} 
Clinical \\
Feature \\
\end{tabular} & Platelet counts & \begin{tabular}{|l|} 
Hormonal \\
Profile
\end{tabular} & $\begin{array}{l}\text { MRI } \\
\text { findings } \\
\end{array}$ & Management & Follow up \\
\hline $\begin{array}{l}\text { Kumar et al } \\
(2011)\end{array}$ & $31 / \mathrm{F}$ & \begin{tabular}{|l|} 
Vision loss, \\
H/A, \\
vomiting
\end{tabular} & \begin{tabular}{|l|} 
Hypothyroid, \\
rest of the \\
hormonal profile \\
normal
\end{tabular} & $\begin{array}{l}\text { Low } \\
(45,000 / \mathrm{m} \\
\mathrm{m} 3)\end{array}$ & 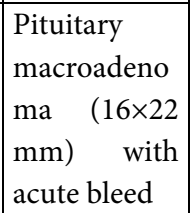 & $\begin{array}{l}\text { Endoscopic } \\
\text { endonasal } \\
\text { decompressio } \\
\mathrm{n} \text { of the } \\
\text { tumor }\end{array}$ & \begin{tabular}{|l|} 
Residual \\
bitemporal \\
field defects at \\
3-month F/U
\end{tabular} \\
\hline $\begin{array}{l}\text { Wildenberg } \\
\text { et al - Case } \\
1(2012)\end{array}$ & $40 / \mathrm{M}$ & \begin{tabular}{|l|}
$\mathrm{H} / \mathrm{A}$, \\
vomiting, \\
acromegali \\
c features
\end{tabular} & $\begin{array}{l}\text { Acromegaly; } \\
\text { Hypogonadotro } \\
\text { pic } \\
\text { hypogonadism }\end{array}$ & \begin{tabular}{|l|} 
Mildly \\
decreased \\
$(98,000 / \mathrm{m}$ \\
$\mathrm{m} 3)$
\end{tabular} & \begin{tabular}{|l|} 
Intrasellar \\
lesion s/o - \\
pituitary \\
apoplexy
\end{tabular} & \begin{tabular}{|l|} 
Transsphenoi \\
dal surgery 20 \\
days after \\
apoplexy
\end{tabular} & \begin{tabular}{|l} 
Acromegaly \\
persists; other \\
hormones \\
normal
\end{tabular} \\
\hline $\begin{array}{l}\text { Wildenberg } \\
\text { et al - Case } \\
2(2012)\end{array}$ & $38 / \mathrm{M}$ & $\begin{array}{l}\text { Prolactino } \\
\text { ma on } \\
\text { medical } \\
\text { Mx, } \\
\text { presents } \\
\text { with visual } \\
\text { field } \\
\text { deficits }\end{array}$ & \begin{tabular}{|l} 
Hypogonadism; \\
Prolactin \\
decreased on \\
medical \\
management
\end{tabular} & \begin{tabular}{|l} 
Mildly \\
decreased \\
$(79,000 / \mathrm{m}$ \\
$\mathrm{m} 3)$
\end{tabular} & 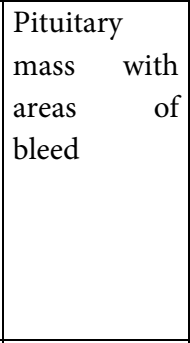 & $\begin{array}{l}\text { Urgent } \\
\text { transsphenoi } \\
\text { dal surgery }\end{array}$ & $\begin{array}{l}\text { Visual field } \\
\text { defects } \\
\text { improved. } \\
\text { Hormonal } \\
\text { deficits persist }\end{array}$ \\
\hline $\begin{array}{l}\text { Tan et al. } \\
(2014)\end{array}$ & $53 / \mathrm{M}$ & $\begin{array}{l}\text { Acute-onse } \\
t \text { Lt third, } \\
\text { sixth nerve } \\
\text { palsy, Rt } \\
\text { temporal } \\
\text { hemianopi } \\
\text { a }\end{array}$ & $\begin{array}{l}\text { Hyperprolactino } \\
\text { ma; thyrotropin } \\
\text { and } \\
\text { gonadotropin } \\
\text { deficiency }\end{array}$ & $\begin{array}{l}\text { Low } \\
(16,000 / \mathrm{m} \\
\mathrm{m} 3)\end{array}$ & \begin{tabular}{|l|}
$37 \times 24 \times 31$ \\
mm \\
hemorrhagic \\
pituitary \\
adenoma \\
with B/L \\
cavernous \\
sinus \\
extension
\end{tabular} & $\begin{array}{l}\text { Underwent } \\
\text { transsphenoi } \\
\text { dal surgery } \\
\text { for } \\
\text { decompressio } \\
\text { n (two } \\
\text { surgeries) }\end{array}$ & \begin{tabular}{|lr} 
Prolactin & \\
normal, & \\
anterior & \\
pituitary & \\
deficits and \\
right eye \\
visual defects \\
persist
\end{tabular} \\
\hline $\begin{array}{l}\text { Mishra et al } \\
(2015)\end{array}$ & $43 / \mathrm{M}$ & $\begin{array}{l}\text { Decreased } \\
\text { vision, } \\
\text { H/A, } \\
\text { vomiting }\end{array}$ & $\begin{array}{l}\text { Normal } \\
\text { hormonal profile }\end{array}$ & $\begin{array}{l}\text { Low } \\
(47,000 / \mathrm{m} \\
\mathrm{m} 3)\end{array}$ & \begin{tabular}{|ll|}
\multicolumn{2}{|l}{$23 \times 21 \times 20$} \\
mm $\quad$ sellar \\
and \\
suprasellar \\
mass $\quad$ with \\
bleed &
\end{tabular} & $\begin{array}{l}\text { Urgent } \\
\text { transsphenoi } \\
\text { dal } \\
\text { decompressio } \\
\mathrm{n} \text { of tumor }\end{array}$ & $\begin{array}{l}\text { Visual acuity } \\
\text { improved. } \\
\text { Residual } \\
\text { visual field } \\
\text { deficits persist }\end{array}$ \\
\hline $\begin{array}{l}\text { Balaparames } \\
\text { wara Rao et } \\
\text { al (2016) }\end{array}$ & $45 / \mathrm{M}$ & \begin{tabular}{|l} 
Severe \\
$\mathrm{H} / \mathrm{A}$, \\
vomiting \\
and LOC
\end{tabular} & $\begin{array}{l}\text { Normal } \\
\text { hormonal profile }\end{array}$ & $\begin{array}{l}\text { Low } \\
(27,000 / \mathrm{m} \\
\mathrm{m} 3)\end{array}$ & \begin{tabular}{|l} 
Pituitary \\
apoplexy \\
(sellar and \\
suprasellar \\
lesion) and \\
obstructive \\
hydrocephal
\end{tabular} & $\begin{array}{l}\text { EVDs to } \\
\text { manage HCP; } \\
\text { followed by } \\
\text { endoscopic } \\
\text { endonasal } \\
\text { decompressio } \\
n\end{array}$ & \begin{tabular}{|l} 
Vision \\
normal. \\
Anterior \\
pituitary \\
deficits \\
present
\end{tabular} \\
\hline
\end{tabular}




\begin{tabular}{|c|c|c|c|c|c|c|c|}
\hline & & & & & us & & \\
\hline $\begin{array}{l}\text { Varma et al } \\
(2016)\end{array}$ & $39 / \mathrm{F}$ & \begin{tabular}{|l|} 
Fever, \\
melena, \\
vision loss \\
,altered \\
sensorium
\end{tabular} & $\begin{array}{l}\text { Hypothyroid, } \\
\text { Hypogonadotro } \\
\text { phic } \\
\text { hypogonadism }\end{array}$ & $\begin{array}{l}\text { Low } \\
(11,000 / \mathrm{m} \\
\mathrm{m} 3)\end{array}$ & \begin{tabular}{|l} 
Pituitary \\
adenoma \\
with \\
apoplexy
\end{tabular} & $\begin{array}{l}\text { Urgent } \\
\text { Decompresio } \\
\text { n of Tumor }\end{array}$ & $\begin{array}{l}\text { Symptoms } \\
\text { Subsided }\end{array}$ \\
\hline Our Case & $48 / \mathrm{F}$ & \begin{tabular}{|l} 
Fever, \\
H/A, \\
Vision \\
Loss
\end{tabular} & $\begin{array}{l}\text { Hypothyroidism } \\
\& \\
\text { Hypoprolactino } \\
\text { ma }\end{array}$ & $\begin{array}{l}\text { Low } \\
(17,000)\end{array}$ & \begin{tabular}{|l|} 
Pituitary \\
apoplexy \\
(sellar and \\
suprasellar \\
lesion)
\end{tabular} & $\begin{array}{l}\text { Urgent } \\
\text { transsphenoi } \\
\text { dal } \\
\text { decompressio } \\
\mathrm{n} \text { of tumor }\end{array}$ & $\begin{array}{l}\text { Visual acuity } \\
\text { improved, } \\
\text { hypocortisole } \\
\text { mia }\end{array}$ \\
\hline
\end{tabular}

In our case we had a smooth outcome but as reported by Balaparameswara Rao et al, it can be strormy i.e. Fluid \& electrolyte imbalance which can occur in pituitary surgery. In all cases Decompresion of the tumour by transpenoidal technique was done and was successful. Vision improved in half of the cases.

\section{Conclusions}

Pituitary apoplexy can occur in setting of Dengue hemorrhagic fever. Dengue hemorrhagic fever should be considered as a cause of pituitary apoplexy. Transsphenoidal decompression of the tumor has good results in these cases. If the diagnosis is made in time, urgent treatment in the form of decompression of optic nerves may help to save vision.

\section{Correspondence}

Dr. Abhay Singh - Fortis Hospital, Sec-62, Gautambudh Nagar, Noida, U.P. India - 201301

Email-abhaysingh291183@gmail.com

Mobile No. 8130503451

\section{References}

1. Möller Goede DL, Brändle M, Landau K, Bernays RL, Schmid C. Pituitary apoplexy: Re evaluation of risk factors for bleeding into pituitary adenomas and impact on outcome. Eur J Endocrinol 2011; 164:37 43.

2. Semple PL, Webb MK, de Villiers JC, Laws ER Jr. Pituitary apoplexy. Neurosurgery 2005; 56: 6572.

3. Wiwanitkit V. Dengue fever: Diagnosis and treatment. Expert Rev Anti Infect Ther 2010; 8:841-5.

4. Simmons CP, Farrar JJ, Nguyen VV, Wills B. Dengue. N Engl J Med 2012; 366:1423 32.

5. Biousse V, Newman NJ, Oyesiku NM. Precipitating factors in pituitary apoplexy. J Neurol Neurosurg Psychiatry 2001; 71:542 5 .

6. Balaparameswara Rao SJ, Savardekar AR, Nandeesh $\mathrm{BN}$, Arivazhagan A. Management dilemmas in a rare case of pituitary apoplexy in the setting of dengue hemorrhagic fever. Surg Neurol Int 2017;8:4. 
7. Arafah BM, Taylor HC, Salazar R, Saadi H, Selman WR. Apoplexy of a pituitary adenoma after dynamic testing with gonadotropin releasing hormone. Am J Med 1989; 87:103 5 .

8. Kumar V, Kataria R, Mehta VS. Dengue hemorrhagic fever: A rare cause of pituitary tumor hemorrhage and reversible vision loss. Indian J Ophthalmol 2011; 59:311 2.

9. Mishra SS, Panigrahi S, Das S. Dengue hemorrhagic fever: A rare case of pituitary apoplexy. Neurol India 2014; 62:92 3 .

10. Tan SK, Seow CJ, Tan E, Chau YP, Dalan R. Pituitary apoplexy secondary to thrombocytopenia due to dengue hemorrhagic Fever: A case report and review of the literature. Endocr Pract 2014;20:58 64

11. Wildemberg LE, Neto LV, Niemeyer P, Gasparetto EL, Chimelli L, Gadelha MR. Association of dengue hemorrhagic fever with multiple risk factors for pituitary apoplexy. Endocr Pract 2012; 18:97 101.

12. Varma, Harsha SH, Reddy, Amaresh P, Pradeep. A rare case of pituitary apoplexy secondary to dengue haemorrhagic fever. Indian J Endocrino \& Metabol 2016; 21:p46-46. 1p.

13. Sanjay S, Wagle AM, Au Eong KG. Optic neuropathy associated with dengue fever. Eye. 2008; 22:722-4. 